\title{
Experimental stand controlled by web interface for data acquisition in punching process
}

\section{Stelian UȚULEANU ${ }^{1, a^{*}}$, Aurelian VLASE $2, b$, Gheorghe SINDILÄ $\breve{A}^{3, c}$ and Nicolae CĂPĂȚÂNĂ ${ }^{4, d}$}

\author{
1, 2, 3, 4University POLITEHNICA of Bucharest, Splaiul Independentei no. 313, District 6, Romania \\ a*stelian_u_86@yahoo.com, baurelvlase@yahoo.com, 'sindila@yahoo.com, \\ dnicu_capatana26@yahoo.com
}

Keywords: data aquisition, punching, web interface, LabVIEW.

\begin{abstract}
Virtual Instrumentation" is the realization of instrumentation, analysis and data processing using a computer. The control of experiments through the web interface provides greater flexibility to the measurement, greater control over results and not least a better processing of the obtained data. There has been made virtual laboratories controlled by web interface for various specialties, but have not been made for the punching process.
\end{abstract}

\section{Introduction}

The virtual instrumentation concept is the realization of instrumentation, analysis and data processing using a computer. Extending at large-scale of computer networks greatly simplifies the monitoring and control processes. Programming environments such as LabVIEW, Measurement Studio, Lookout Bridgeview or Lookout have a variety of functions for data transmission through specific Internet protocols [1].

The prestigious international universities were created labs with access through the Internet. For this purpose there are presented the following [2,3]:

- $\quad$ at the Stanford University (California, USA), students can connect to the optical laboratory to perform an experiment to measure the physical properties of a laser diode.

- at the Swiss Federal Institute of Technology (Zurich, Switzerland) during the course the students watch how the teacher controls a mechanical system using a portable computer and they will study the experiment in detail, at home, with the watching possibility of the video recording of experiment.

- at the Dalhousie University (Halifa - Nova Scotia, Canada), students can connect to the Virtual Laser Lab, 24 hours / day, 7 days / week, and can achieve up to 10 experiments.

- at the University of Maribor (Maribor, Slovenia), students have a computer lab with a network managed by a server. Each student has a folder on the server, which is updated after the experiment.

\section{Materials and experimental procedure}

Data acquisition system consists of mechanical press, force transducer, course transducer, one conditioner for each channel, data acquisition board, server, the local area network (or Internet) and the computer from which is accessed the measuring stand [fig. 1]. The data acquisition board used is NI-USB-6008, its configuration for input signals was made on two analog channels and for output signals its configuration was made on an analogue channel. Acquisition is carried out at a frequency of $5 \mathrm{kHz}$ and 3,000 entries, resulting an acquisition time of 0.6 seconds $[4,5]$.

To control the mechanical press was added a manual switch SW1 [fig. 2] (switching between automatic mode and manual mode of the press operation) and was used a relay made by OMRON, G3NA-205B model, that connects the acquisition board and the electrical circuit of the press.

A schematic representation of the data acquisition stand is shown in fig. 1 


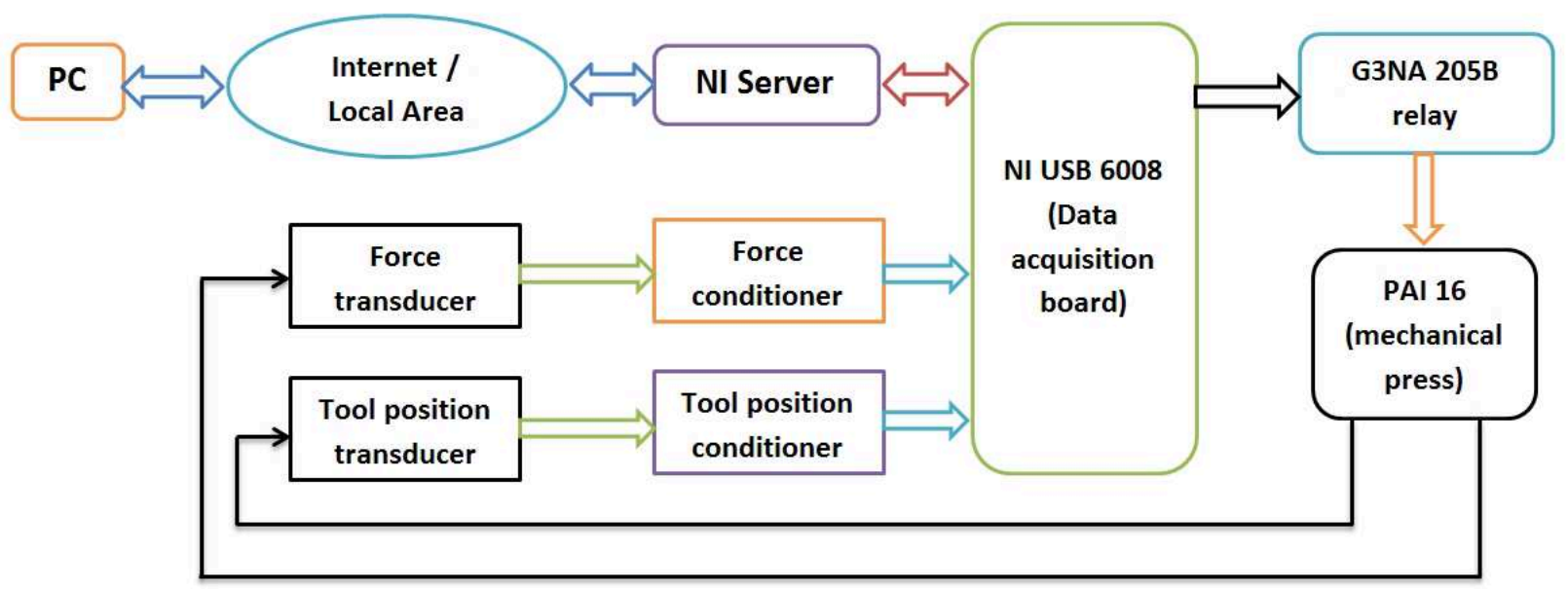

Fig. 1 The schematic representation of the data acquisition stand

The electrical circuit added for automatic operation mode for mechanical press is presented in fig. 2. The G3NA-205B relay is cool down using an aluminum radiator and a 12 VCC ventilator.

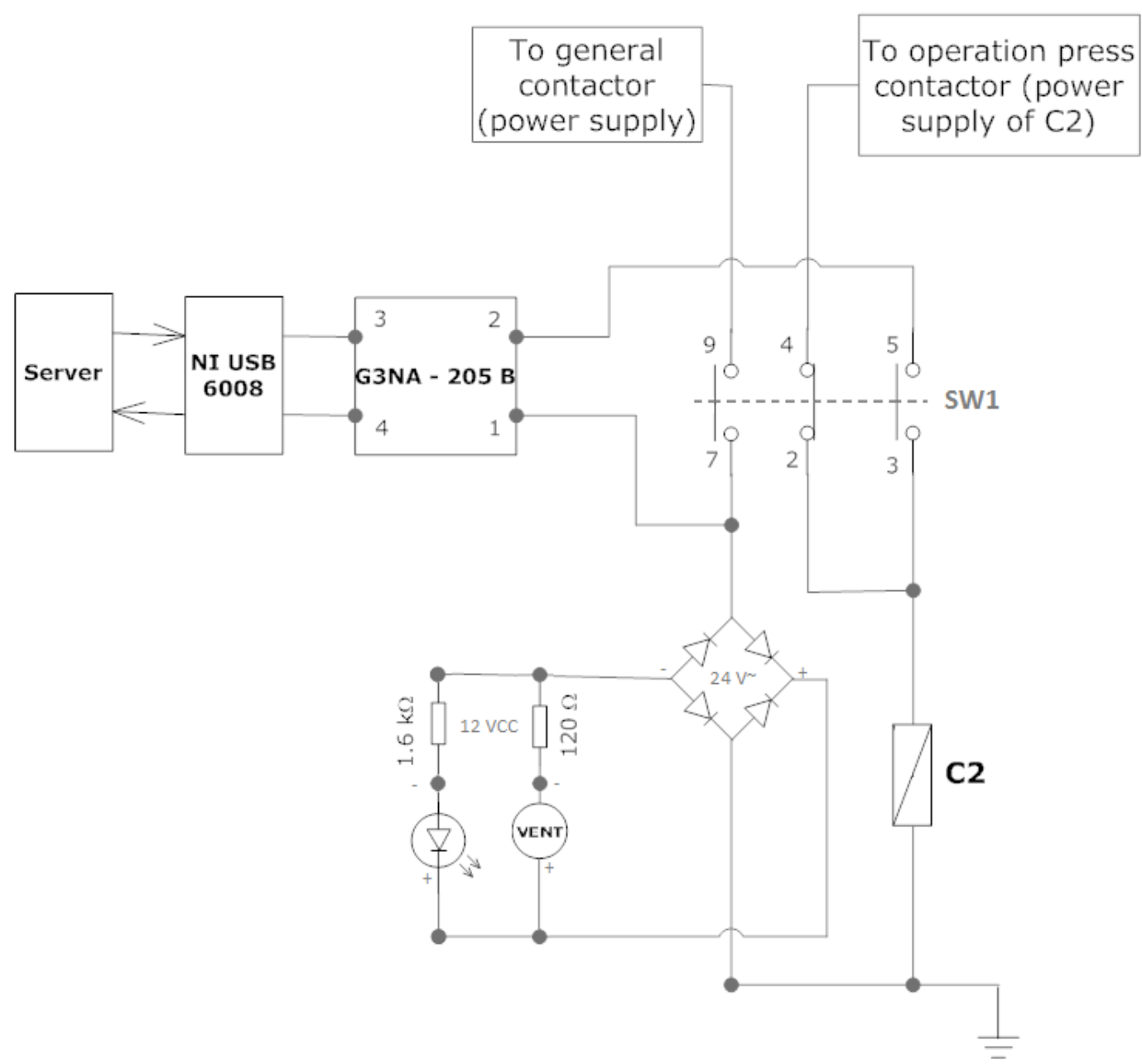

Fig. 2 The electrical circuit added for automatic operation mode for mechanical press

The acquisition program is created in LabVIEW 2012, x64 version, and its structure has one button to stop the acquisition - "Stop", two numerical controls, for constant of the force conditioner - "Force constant" and for course conditioner - "Course constant", an XY chart - "Course - Force Graph", where the force variation is observed according to the movement of the punch tool and two graphs for observing the variation of force and displacement according to time. Also, there are four 
indicators for "Min Force", "Max Force", "Min Course" and "Max Course" where are presented the minimum and maximum values of force and course after the end of data acquisition.

The program's diagram is presented in fig. 3. To protect the system resources it was added a delay of 0.1 seconds. Was used a "Flat Sequence Structure" to generate $5 \mathrm{~V}$ output, maintain the voltage for 0.2 seconds and stop the output signal which control the relay.

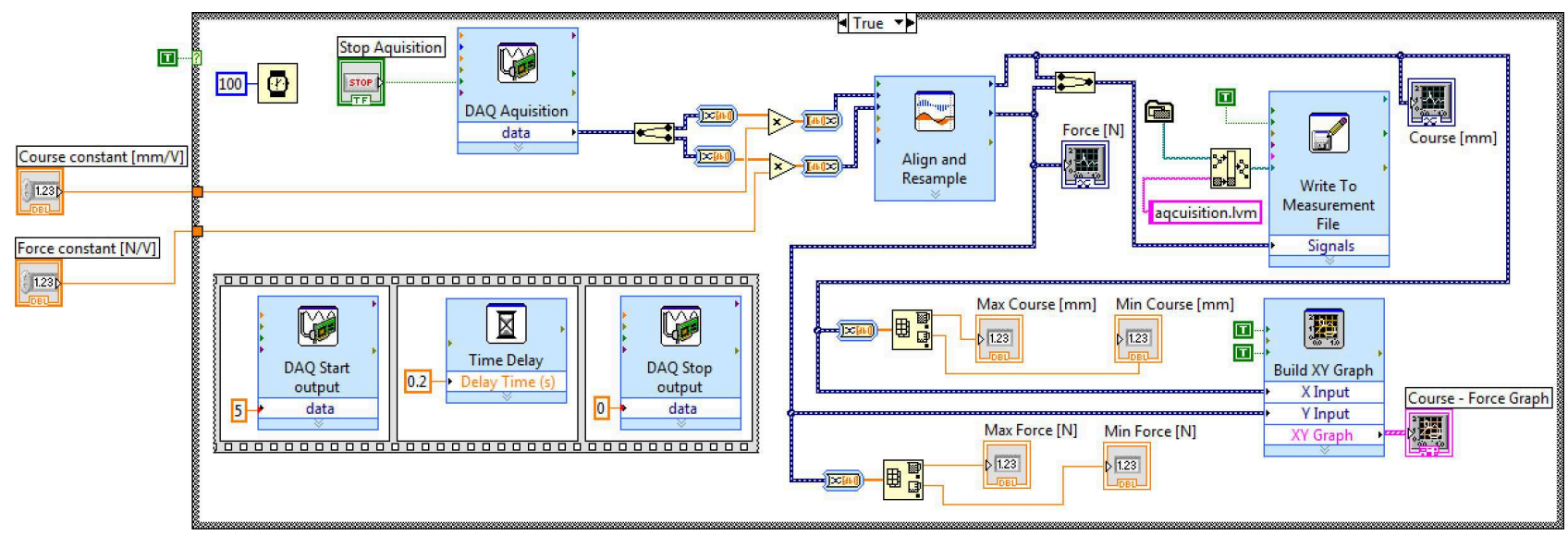

Fig. 3 The data acquisition program's diagram

The acquisition data is split in two channels and each sample is corrected with the constant value (course and force constant). After that the signals are filtered using the "Align and Resample". Now we can use the to determine the minimum and maximum values of force and course, to create the "Course - Force Graph" and graphs for variation of force and course according to time.

Before saving the acquisition data, it's necessary to merge the signals. The combined signal is use by "Write To Measurement File" to create the file with obtained data of the experiment.

The easiest way to control the front panel of a LabVIEW program is to access the "Web Publishing Tool” from "Tools" section [fig. 4].

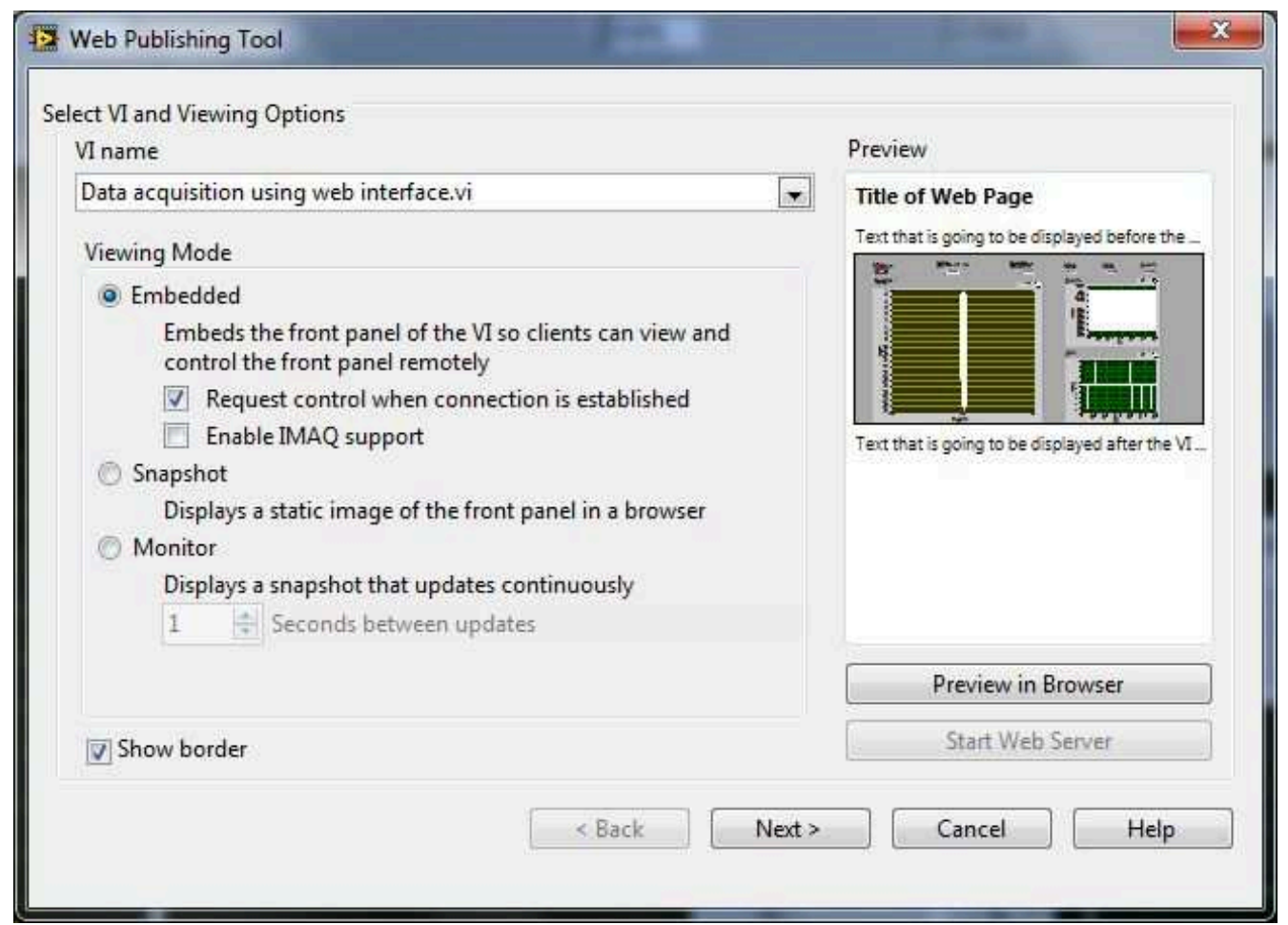

Fig. 4 Web Publishing Tool pop-up window 
In the pop-up window that appears [fig. 4] is select the VI that will be controlled through web interface. Also, is necessary to check the "Request control when connection is established" option to have the control over the front panel.

The next step is to add the document title, header and footer of the web page [fig. 5]. The text is only informative and can be inserted what you want for a better description of application.

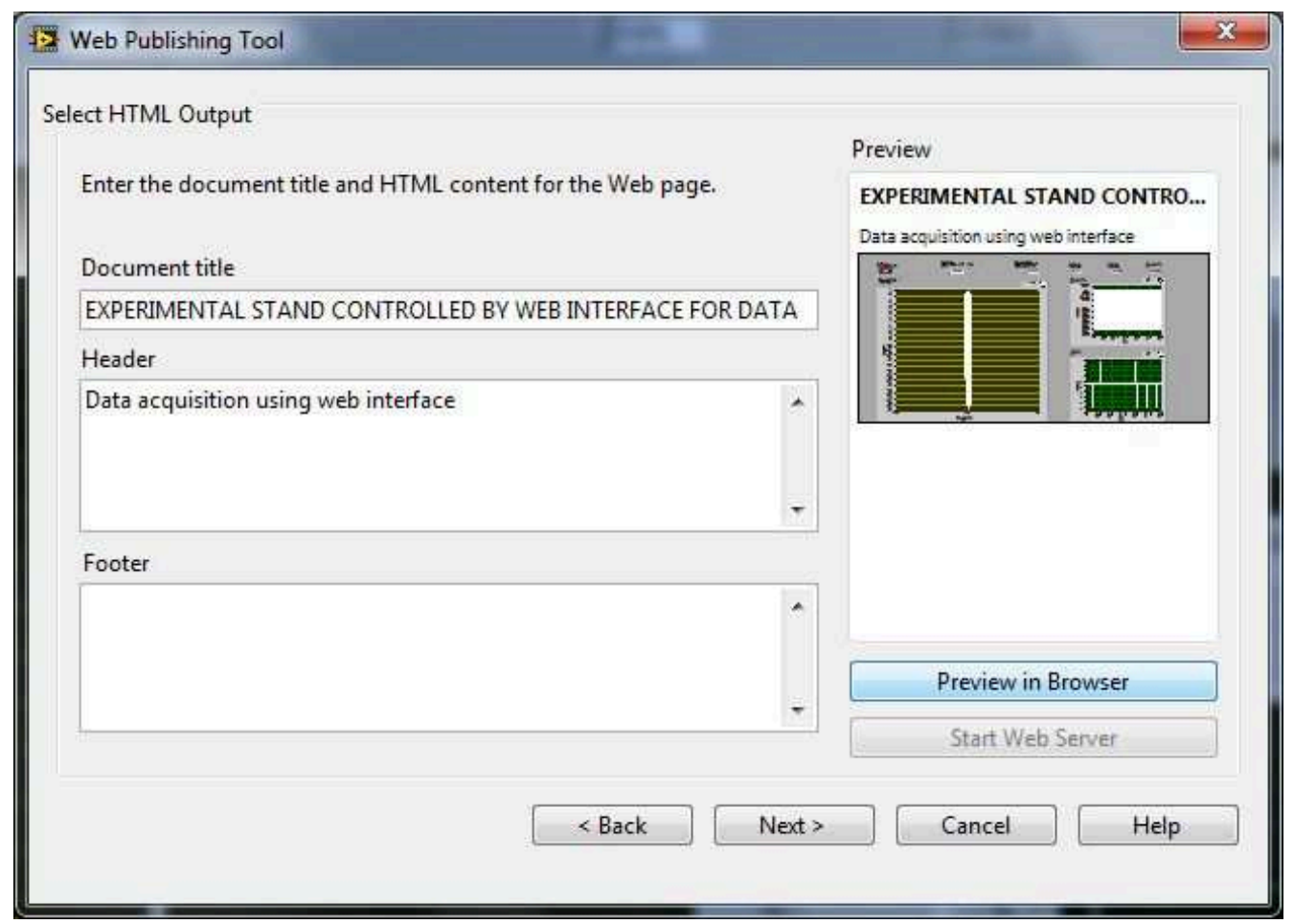

Fig. 5 Web Publishing Tool - HMTL Output pop-up window

Forwards [fig. 6] should be selected the destination folder where the web page will be saved, must be set the filename and will be displayed the accessing address of the new created web page.

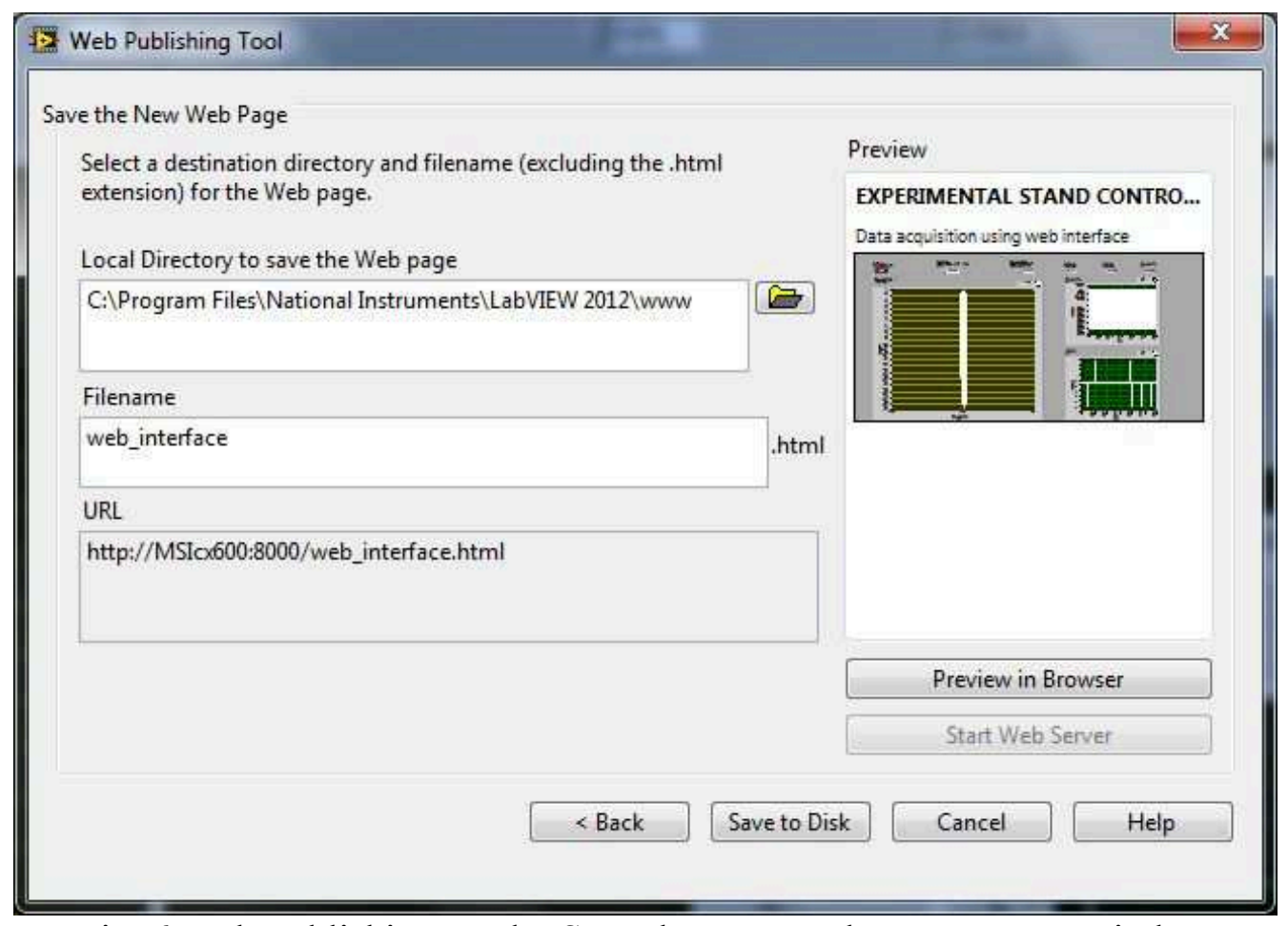

Fig. 6 Web Publishing Tool - Save the New Web Page pop-up window 
In the end of web page creation will be displayed a window with the page's URL and exists the possibility of connection to this address [fig. 7].

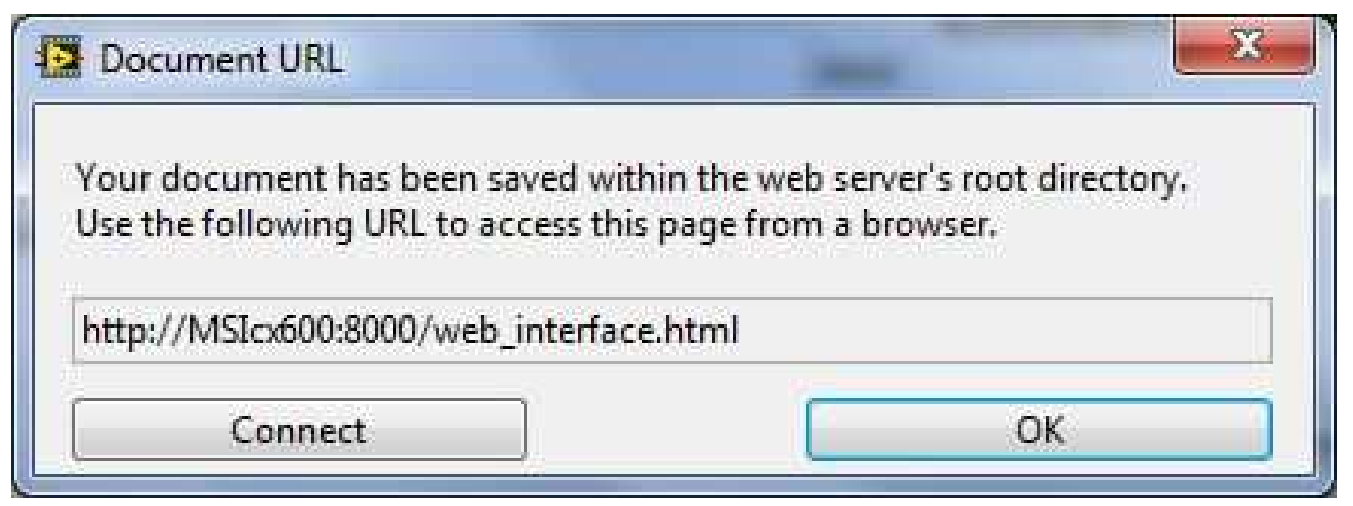

Fig. 7 The Document URL pop-up window for the new web page

It is necessary to install some plugins for web browsers to display the front panel of a VI.

In this case was installed the "LVRTE2012f3min" package downloaded from National Instruments web page [6]. After the installation is complete the computer should be restarted.

The Mozilla Firefox web browser should be set to allow the Remote Front Panels (RFP) plugins by changing the configuration. To do this, enter "about:config" in the URL bar and scroll down to "plugins.load_appdir_plugins". Double-click on this row to change the value from false to true. Restart Firefox and the RFP plugin should now appear in the plugins list and the application should be viewable in browser. To check the list of installed plugins should be typed "about:plugins" in the URL bar. [7].

\section{Results and discussions}

During the experiments were obtained encouraging results on the use of control experiments using the web interface of the laboratory work associated with data acquisition in punching process.

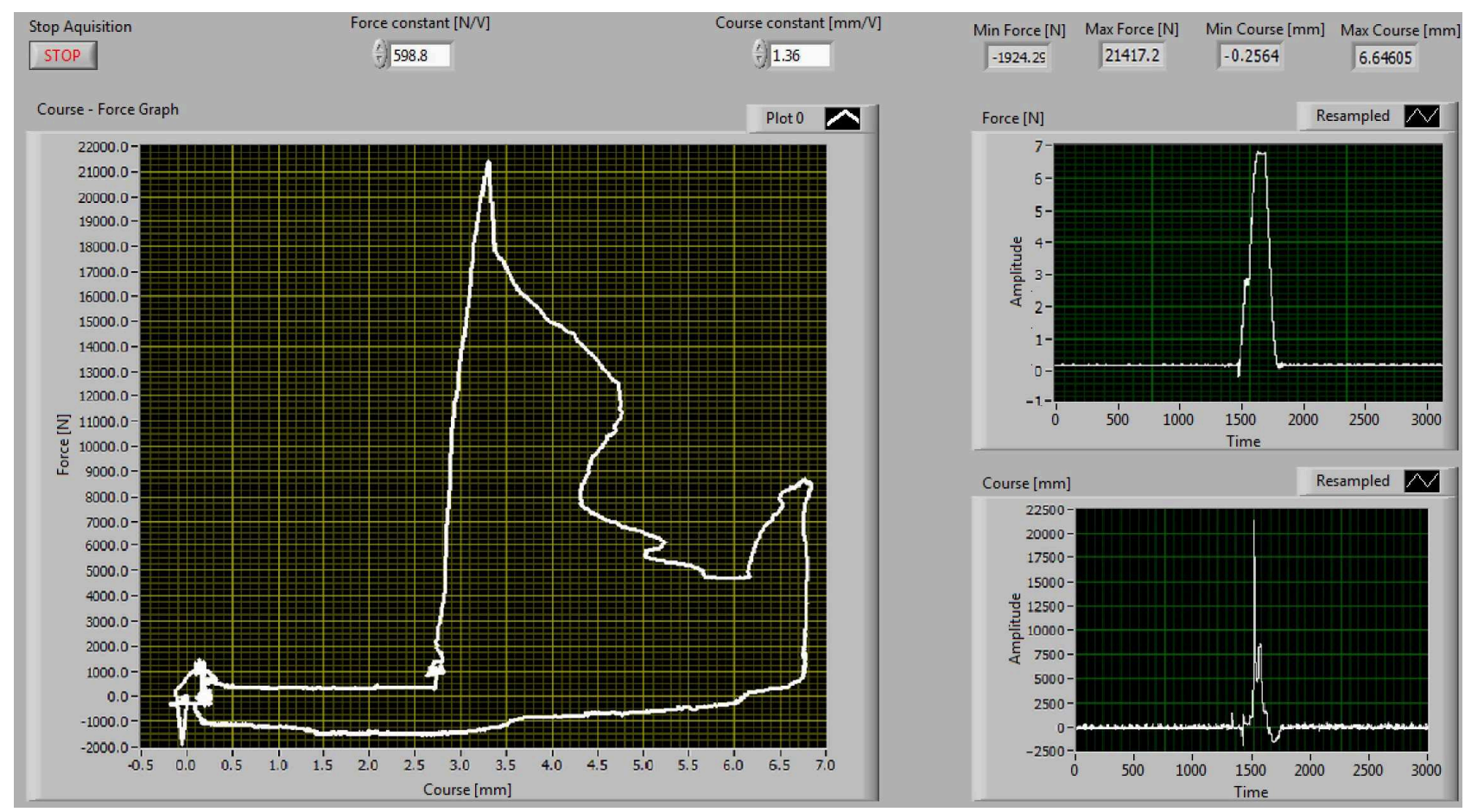

Fig. 8 Results of data acquisition in the application's front panel 
This technology provides greater flexibility to the measurement of forces, a better control of the obtained results and a better processing of the obtained data.

Figure 8 presents the results of data acquisition for a triangular shape with $30 \mathrm{~mm}$ perimeter, made of AISI 304 material and having a thickness of $0.51 \mathrm{~mm}$.

\section{Conclusions}

Although has been made virtual laboratories controlled by web interface for various specialties, but have not been made for the punching process. This paper tries to implement this technology in cold plastic deformation processes.

This technology should be implemented in education systems because offers flexibility, security, reliability and is a simple and good solution for academic research.

In further research will be analyzed the influence of rolling direction (at different angles: $0^{\circ}, 15$ $\circ, 30^{\circ}, 45^{\circ}, 60^{\circ}, 75^{\circ}$ and $90^{\circ}$ ) on the punching force and the development of data acquisition program via web interface for extrusion process.

Also, will be improved the web control using a login sesisson base on a user name and a password for each student created by the network administrator, will be added a webcam to see in real time the experiment and the network will be managed by a server to allow each student to have a folder on the server, which is updated after the experiment.

\section{References}

[1] E. Luncă, C. Donciu, M. Crețu, Aplicații server - client în LabVIEW (I). Comunicația TCP-IP și Remote Device Access, Magazine of Virtual Instrumentation, 2004 (in Romanian).

[2] Ph. Krauss, S. Günther, M. Backmeyer, Standard Tools for Reomte Learning. DistanceLearning Remote Laboratories using LabVIEW, Magazine of Virtual Instrumentation, 2004.

[3] Bojan, G., Darko, H., Ladislav, M., Vojko, M., Using the Internet and Virtual Instrumentation to enhace the learning of Electrical Measurements, Magazine of Virtual Instrumentation, 2004.

[4] S. Uțuleanu, The influence of punched contour geometry over the punch force, International Scientific Conference on Advances in Mechanical Engineering, 10-11 October 2013, Debrecen, Hungary.

[5] National Instruments, (2012) LabVIEW 2012 Help.

[6] Information on http://www.ni.com/download/labview-run-time-engine-2012/3432/en/.

[7] Information on http://digital.ni.com/public.nsf/allkb/CF91AE26198992A686257B79006959C0. 